\title{
A fixed point theorem for discontinuous functions
}

Citation for published version (APA):

Herings, P. J. J., van der Laan, G., Talman, A. J. J., \& Yang, Z. (2005). A fixed point theorem for discontinuous functions. METEOR, Maastricht University School of Business and Economics. METEOR Research Memorandum No. 010 https://doi.org/10.26481/umamet.2005010

Document status and date:

Published: 01/01/2005

DOI:

10.26481/umamet.2005010

Document Version:

Publisher's PDF, also known as Version of record

\section{Please check the document version of this publication:}

- A submitted manuscript is the version of the article upon submission and before peer-review. There can be important differences between the submitted version and the official published version of record.

People interested in the research are advised to contact the author for the final version of the publication, or visit the DOI to the publisher's website.

- The final author version and the galley proof are versions of the publication after peer review.

- The final published version features the final layout of the paper including the volume, issue and page numbers.

Link to publication

\footnotetext{
General rights rights.

- You may freely distribute the URL identifying the publication in the public portal. please follow below link for the End User Agreement:

www.umlib.nl/taverne-license

Take down policy

If you believe that this document breaches copyright please contact us at:

repository@maastrichtuniversity.nl

providing details and we will investigate your claim.
}

Copyright and moral rights for the publications made accessible in the public portal are retained by the authors and/or other copyright owners and it is a condition of accessing publications that users recognise and abide by the legal requirements associated with these

- Users may download and print one copy of any publication from the public portal for the purpose of private study or research.

- You may not further distribute the material or use it for any profit-making activity or commercial gain

If the publication is distributed under the terms of Article $25 \mathrm{fa}$ of the Dutch Copyright Act, indicated by the "Taverne" license above, 


\title{
A Fixed Point Theorem for Discontinuous Functions ${ }^{1}$
}

\author{
Jean-Jacques Herings, ${ }^{2}$ Gerard van der Laan, ${ }^{3}$ Dolf Talman, ${ }^{4}$ Zaifu Yang ${ }^{5}$
}

December 16, 2004

\footnotetext{
${ }^{1}$ This research was carried out while Jean-Jacques Herings, Gerard van der Laan and Zaifu Yang were visiting the CentER for Economic Research, Tilburg University. The visit of Zaifu Yang has been made possible by financial support of CentER and the Netherlands Organization for Scientific Research (NWO). Jean-Jacques Herings would like to thank the Netherlands Organisation for Scientific Research (NWO) for financial support.

${ }^{2}$ P.J.J. Herings, Department of Economics, Maastricht University, P.O. Box 616, 6200 MD Maastricht, The Netherlands, p.herings@algec.unimaas.nl

${ }^{3}$ G. van der Laan, Department of Econometrics and Tinbergen Institute, Vrije Universiteit, De Boelelaan 1105, 1081 HV Amsterdam, The Netherlands, glaan@feweb.vu.nl

${ }^{4}$ A.J.J. Talman, Department of Econometrics \& Operations Research and CentER, Tilburg University, P.O. Box 90153, 5000 LE Tilburg, The Netherlands. E-mail: talman@uvt.nl

${ }^{5}$ Z. Yang, Faculty of Business Administration, Yokohama National University, Yokohama 2408501, Japan. E-mail: zyang@business.ynu.ac.jp
} 


\begin{abstract}
In this paper we prove the following fixed point theorem. Consider a non-empty bounded polyhedron $P$ and a function $f: P \rightarrow P$ such that for every $x \in P$ for which $f(x) \neq x$ there exists $\delta>0$ such that for all $y, z \in B(x, \delta) \cap P$ it holds that

$$
(f(y)-y)^{\top}(f(z)-z) \geq 0,
$$

where $B(x, \delta)$ is the ball in $\mathbb{R}^{n}$ centered at $x$ with radius $\delta$. Then $f$ has a fixed point, i.e., there exists a point $x^{*} \in P$ satisfying $f\left(x^{*}\right)=x^{*}$. The condition allows for various discontinuities and irregularities of the function. In case $f$ is a continuous function, the condition is automatically satisfied and thus the Brouwer fixed point theorem is implied by the result. We illustrate that a function that satisfies the condition is not necessarily upper or lower semi-continuous. A game-theoretic application is also discussed.
\end{abstract}

Keywords: Fixed point, simplicial subdivision, discontinuity, equilibrium.

AMS subject classifications: Primary, 54H25, 65K10; Secondary, 49J53, 68W25.

JEL classifications: C62, C63. 


\section{Introduction}

Almost one century ago Brouwer (1912) proved a remarkable result saying that any continuous function from the $m$-dimensional unit ball to itself has a fixed point, a point that is mapped by the function into itself. The Brouwer fixed point theorem was one of the early major achievements of algebraic topology. This celebrated theorem has been generalized in several ways, for general surveys of the literature see, e.g., Smart (1974) and Istratescu (1981). For instance, Schauder (1930) generalized the theorem to Banach spaces. Kakutani (1942) gave an extension to upper semi-continuous point-to-set mappings.

Existence results of fixed points in case the function is not continuous were given by Tarski (1955) and Caristi (1976). Tarski's theorem is restricted to functions on a sublattice satisfying some monotonicity condition. Caristi's theorem concerns functions satisfying a non-expansion condition. However, it should be noticed that both these theorems do not cover Brouwer's theorem, because a continuous function does not need to satisfy the conditions in Tarski's or Caristi's theorem. Another major development during the last few decades is about the computation of fixed points of a continuous function or upper semi-continuous point-to-set mapping, see, e.g., Scarf (1973), Todd (1976), Allgower and Georg (1990), and Yang (1999).

Nowadays, the Brouwer, Kakutani, and Tarski theorems have become the most often used tools in economics and game theory, see, e.g., Arrow and Hahn (1971), Fudenberg and Tirole (1991), and Herings (1996). Also on the practical frontier fixed point methods are used by applied economists to analyse equilibrium models, for instance, to study the effects of policy and technical changes, see e.g., Shoven and Whalley (1992).

In this paper, we give a general condition, to be called the locally gross direction preserving property, under which a fixed point of a function from an arbitrary non-empty convex polyhedron to itself exists. The condition says that at any point $x$ not being a fixed point of $f$ it must hold that for any two points $y$ and $z$ in some neighborhood of $x$ the vectors $f(y)-y$ and $f(z)-z$ should point grossly in the same direction, i.e. the inner product of $f(y)-y$ and $f(z)-z$ should be non-negative. Surprisingly, this allows for all kinds of discontinuities and irregularities of the function, even at a fixed point. We show that any continuous function is locally gross direction preserving, so that Brouwer's fixed point theorem is covered by our new theorem. We illustrate that a function that satisfies the condition is not necessarily upper or lower semi-continuous. On the other hand, a function satisfying the monotonicity condition of Tarski does not need to be locally gross direction preserving. To our best knowledge, our new theorem seems to be the first of such kind that both allows discontinuities and covers Brouwer's theorem simultaneously in the single-valued case. Discontinuities arise naturally in various fields of study, such as engineering, economics and game theory. Here we also briefly discuss an application of our 
theorem in game theory.

This paper is organized as follows. Section 2 establishes the theorem and gives some examples to illustrate its generality. Section 3 presents a game theoretic application.

\section{An existence theorem}

Let $P$ be a non-empty bounded polyhedron in the $n$-dimensional Euclidean space $\mathbb{R}^{n}$ and let $f$ be a function from $P$ to $P$. We call $x^{*} \in P$ a fixed point of $f$ if $f\left(x^{*}\right)=x^{*}$, i.e., $f$ maps $x^{*}$ into itself. Brouwer (1912) proved that if $f$ is continuous on $P$, then $f$ has a fixed point. In this paper we provide a fixed point theorem that allows for discontinuities, even at a fixed point itself. More precisely, the existence of a fixed point is guaranteed when $f$ satisfies the so-called locally gross direction preserving property. A discrete version of this property was originally used in Yang (2004) to prove the existence of a fixed point in case the domain is a discrete set. For $x \in \mathbb{R}^{n}$ and $\delta>0$, let $B(x, \delta)$ denote the $n$-dimensional ball in $\mathbb{R}^{n}$ with center $x$ and radius $\delta$.

Definition 2.1 A function $f: P \rightarrow P$ is locally gross direction preserving when for every $x \in P$ for which $f(x) \neq x$, there exists $\delta>0$ satisfying that for every $y$, $z \in B(x, \delta) \cap P$ it holds that

$$
(f(y)-y)^{\top}(f(z)-z) \geq 0 .
$$

Throughout the paper, for $h \in \mathbb{N}$, let $I_{h}$ denote the index set $\{1, \ldots, h\}$. We now state the main result of this paper.

Theorem 2.2 Let $P$ be a non-empty bounded polyhedron in $\mathbb{R}^{n}$ and let the function $f: P \rightarrow P$ satisfy the locally gross direction preserving property. Then $f$ has a fixed point.

Proof: Take a sequence of simplicial subdivisions, $\left(T_{k}\right)_{k \in \mathbb{N}}$, of $P$ with mesh size tending to zero if $k$ goes to infinity, i.e., for each $k \in \mathbb{N}, T_{k}$ is a finite collection of simplices whose union is $P$ and for which the intersection of any two simplices is either empty or a common face of both. Since $P$ is a bounded polyhedron (i.e., a polytope) and therefore the convex hull of a finite number of points in $\mathbb{R}^{n}$, such a sequence exists; see for example Talman and Yamamoto (1989). For $k \in \mathbb{N}$, let $\bar{f}^{k}$ denote the piecewise linear approximation of $f$ with respect to $T_{k}$, i.e., for $x \in P$,

$$
\bar{f}^{k}(x)=\sum_{j=1}^{n+1} \lambda_{j} f\left(x^{j}\right),
$$


where $x^{1}, \ldots, x^{n+1}$ are the vertices of a simplex in $T_{k}$ containing $x$, and $\lambda_{1}, \ldots, \lambda_{n+1}$ are the unique non-negative numbers with sum equal to one satisfying

$$
x=\sum_{j=1}^{n+1} \lambda_{j} x^{j}
$$

Since the function $\bar{f}^{k}$ is piecewise linear and therefore continuous on $P$, the Brouwer fixed point theorem implies that for every $k \in \mathbb{N}$ there exists a fixed point $x^{k}$ of $\bar{f}^{k}$.

Next we consider the sequence of points $\left(x^{k}\right)_{k \in \mathbb{N}}$. Since this sequence is a sequence of points in the compact set $P$, there exists a convergent subsequence. Without loss of generality we assume that the sequence $\left(x^{k}\right)_{k \in \mathbb{N}}$ itself converges to some $x^{*} \in P$. For $k \in \mathbb{N}$, let $\sigma^{k}$ be an $n$-dimensional simplex in $T_{k}$ with vertices $x^{k, 1}, \ldots, x^{k, n+1}$ containing $x^{k}$. Then there exist unique non-negative numbers $\lambda_{j}^{k}, j \in I_{n+1}$, with sum equal to 1 , satisfying

$$
x^{k}=\sum_{j=1}^{n+1} \lambda_{j}^{k} x^{k, j} \text { and } \bar{f}^{k}\left(x^{k}\right)=\sum_{j=1}^{n+1} \lambda_{j}^{k} f\left(x^{k, j}\right) .
$$

If $f\left(x^{*}\right)=x^{*}$, then $x^{*}$ is a fixed point of $f$ and the theorem has been proved. Suppose therefore that $x^{*}$ is not a fixed point of $f$. Then according to the condition of the theorem there exists $\delta^{*}>0$ such that for all $y, z \in B\left(x^{*}, \delta^{*}\right) \cap P$ it holds that

$$
(f(y)-y)^{\top}(f(z)-z) \geq 0 .
$$

Since the sequence $\left(x^{k}\right)_{k \in \mathbb{N}}$ converges to $x^{*}$ and the mesh size of $T_{k}$ converges to zero when $k$ goes to infinity, we obtain that for every $j \in I_{n+1}$ the sequence $\left(x^{k, j}\right)_{k \in \mathbb{N}}$ converges to $x^{*}$. Hence, there exists $k^{*} \in \mathbb{N}$ such that for all $k \geq k^{*}$ it holds that $x^{k, j} \in B\left(x^{*}, \delta^{*}\right) \cap P$ for all $j \in I_{n+1}$ and therefore

$$
\left(f\left(x^{k, i}\right)-x^{k, i}\right)^{\top}\left(f\left(x^{k, j}\right)-x^{k, j}\right) \geq 0,
$$

for all $i, j \in I_{n+1}$. On the other hand, since $\bar{f}^{k}\left(x^{k}\right)=x^{k}$, for all $k \in \mathbb{N}$, we have that

$$
\sum_{j=1}^{n+1} \lambda_{j}^{k}\left(f\left(x^{k, j}\right)-x^{k, j}\right)=0^{n},
$$

where $0^{n}$ is the $n$-dimensional vector of zeros. Fix any $k \geq k^{*}$. Since $\sum_{j=1}^{n+1} \lambda_{j}^{k}=1$, there exists $j^{*} \in I_{n+1}$ satisfying $\lambda_{j^{*}}^{k}>0$. Clearly,

$$
\sum_{j=1}^{n+1} \lambda_{j}^{k}\left(f\left(x^{k, j^{*}}\right)-x^{k, j^{*}}\right)^{\top}\left(f\left(x^{k, j}\right)-x^{k, j}\right)=0 .
$$

Since every term in this summation is non-negative, every term must be zero. So, taking $j=j^{*}$ we obtain that

$$
\left(f\left(x^{k, j^{*}}\right)-x^{k, j^{*}}\right)^{\top}\left(f\left(x^{k, j^{*}}\right)-x^{k, j^{*}}\right)=0,
$$


implying that $f\left(x^{k, j^{*}}\right)=x^{k, j^{*}}$. Hence $x^{k, j^{*}}$ is a fixed point of $f$.

Observe that basically the proof shows two possibilities, namely that the limit point $x^{*}$ of the convergent sequence $\left(x^{k}\right)_{k \in \mathbb{N}}$ is a fixed point, or for $k$ large enough, any simplex in the converging sequence has at least one of its vertices as a fixed point. This also implies that the result does not follow from applying the Kakutani fixed point theorem to the mapping $F$ defined as the convex closure of $f$. By definition of the convex closure, $F$ is upper semi-continuous and thus there exists a point $x^{*}$ satisfying $x^{*} \in F\left(x^{*}\right)$. However, a fixed point of the convex closure $F$ of $f$ is not necessarily a fixed point of $f$. This can be seen from the proof, since due to possible discontinuities of $f$, the limit point $x^{*}$ of the converging sequence $\left(x^{k}\right)_{k \in \mathbb{N}}$ is not necessarily a fixed point of $f$, whereas in case of applying the simplicial procedure to the upper semi-continuous convex closure $F$, the limit point $x^{*}$ always is a fixed point of $F$. As a consequence, Theorem 2.2 is not an application of Kakutani fixed point theorem. This can be also understood by observing that Kakutani fixed point theorem generalizes Brouwer fixed point theorem only when the mapping is multi-valued, and it is identical to Brouwer's theorem when the mapping is single-valued; in contrast, our Theorem 2.2 is a generalization of Brouwer's theorem. Indeed, a continuous function is locally gross direction preserving. Hence Theorem 2.2 implies that any continuous function from $P$ to $P$ has a fixed point, the Brouwer fixed point theorem. This gives the following corollary.

Corollary 2.3 Let $P$ be a non-empty bounded polyhedron in $\mathbb{R}^{n}$ and let $f: P \rightarrow P$ be a continuous function. Then $f$ has a fixed point in $P$.

Proof: Take any point $x$ in $P$ and suppose that $f(x) \neq x$. Clearly, $(f(x)-x)^{\top}(f(x)-x)>$ 0 . Consider the function $g: P \times P \rightarrow \mathbb{R}^{n} \times \mathbb{R}^{n}$ defined by

$$
g(y, z)=(f(y)-y)^{\top}(f(z)-z) .
$$

Since $f$ is continuous, $g$ is continuous, so there exists $\delta>0$ so that for all $y, z \in B(x, \delta) \cap P$ it holds that $g(y, z)>0$. Hence $f$ is locally gross direction preserving and the result follows from Theorem 2.2.

To illustrate and examine the locally gross direction preserving property, let us consider the one-dimensional case, i.e. $f:[0,1] \rightarrow[0,1]$. In this case we can interpret the function as the description of an object's movement in time-distance space. Let $t \in[0,1]$ denote time and $f(t) \in[0,1]$ the position of the object at time $t$. This object could be physical, non-physical or imaginary. We could for example think of the movement of a star or a particle through space or the fluctuation of a stock price during time period $[0,1]$. Any fixed point of $f$ represents a time-position combination $(t, f(t))$ on the diagonal in the 
two-dimensional square $[0,1] \times[0,1]$. The locally gross direction preserving property says that, when at time $t$ the object does not lie on the diagonal, then there exists a time span around $t$ in which the object lies on the same side of the diagonal. If this holds, then the theorem states that there exists at least one time-position combination on the diagonal. In particular, in the physical world the theorem demonstrates that no matter how slowly or quickly, and no matter how irregularly or regularly the object moves, it must hit the diagonal at least once during its movement from time 0 to time 1.

A function $f:[0,1] \rightarrow[0,1]$ is said to be lower semi-continuous if for all sequences $\left(x^{k}\right)_{k \in \mathbb{N}}$ with $x^{k} \in[0,1]$ for all $k \in \mathbb{N}$, such that $x^{k} \rightarrow \bar{x}$ it holds that $\liminf _{k \rightarrow \infty} f\left(x^{k}\right) \geq$ $f(\bar{x})$. A function $f:[0,1] \rightarrow[0,1]$ is said to be upper semi-continuous if for all sequences $\left(x^{k}\right)_{k \in \mathbb{N}}$ with $x^{k} \in[0,1]$ for all $k \in \mathbb{N}$, such that $x^{k} \rightarrow \bar{x}$ it holds that $\lim _{\sup _{k \rightarrow \infty}} f\left(x^{k}\right) \leq$ $f(\bar{x})$. Notice that Theorem 2.2 allows for various kinds of discontinuities of the function $f$. It is clear from the example that the function $f$ does not need to be lower semi-continuous or upper semi-continuous.

Although the local gross direction preserving property allows for all kinds of discontinuities, the property puts sufficient conditions to guarantee the existence of a fixed point. This is clarified in the next example of a one-dimensional function on [0,2], where at given point $x, f^{-}(x)$ is the (lower) limit of $f$ from the left and $f^{+}(x)$ the (upper) limit of $f$ from the right.

Example 2.4 Let $f:[0,2] \rightarrow[0,2]$ be continuous at any $x \in[0,2]$, except at $x=1$. Without loss of generality we assume that $f(x)>x$ for all $x<1$ (otherwise there is a fixed point $x^{*}$ satisfying $\left.x^{*}<1\right)$. So, $f^{-}(1) \geq 1$. Now the locally gross direction preserving condition requires that $f(1) \geq 1$, since $f(1)<1$ contradicts that for every $y$ and $z$ in some $B(1, \delta)$ it holds that $(f(y)-y)^{\top}(f(z)-z) \geq 0$. Further, when $f^{+}(1)>1$, then there exists a fixed point $x^{*}>1$. So, suppose $f^{+}(1) \leq 1$. Then the locally gross direction preserving property requires that either (i) $f(1)=1$ and thus $x^{*}=1$ is a fixed point, or (ii) $f(1)>1$ and there exists some $y, 1<y \leq 2$, such that $f(x) \geq x$ for all $1<x \leq y$. In the latter case there is a fixed point $x^{*}>1$. In particular it may occur that $f(x)=x$ for all $1<x \leq y$, corresponding to the case in the proof that for $k$ large enough any simplex in the converging sequence has at least one of it vertices as a fixed point. Observe that the locally gross direction preserving property excludes that $f(1)>1$ and $f^{+}(1)<1$ and that $f(1)<1$ and $f^{-}(1)>1$.

More generally, the locally gross direction preserving condition requires that if $x$ is not a fixed point of $f$ there exists a neighborhood of $x$ such that for any two points $y$ and $z$ in this neighborhood it holds that the vectors $f(y)-y$ and $f(z)-z$ make a sharp angle with each other or are orthogonal to each other, i.e., the direction of these two vectors is grossly 
preserved. This condition replaces continuity at $x$. If $x$ is a fixed point of $f$ nothing about $f$ around $x$ is required.

Locally gross direction preserving does not require that the function is monotone nondecreasing, which property is required by Tarski's theorem. On the other hand, a function satisfying Tarski's theorem does not need to be locally gross direction preserving. For example, if $f(x)=\frac{1}{3}(x+1)$ for $0 \leq x \leq 1$ and $f(x)=\frac{1}{3}(x+3)$ for $1<x \leq 2$, then $f$ is monotone increasing on the interval $[0,2]$, but $f$ is not locally gross direction preserving at $x=1$.

Finally we wish to stress that Theorem 2.2 is restricted to a bounded polyhedron $P$. Whether locally gross direction preserving is sufficient to guarantee the existence of a fixed point on an arbitrary non-empty convex and compact set is still an open question. The problem is that the proof makes use of a simplicial subdivision of $P$, which requires the set to be a polyhedron. Although any arbitrary convex and compact set $C$ can be approximated by a sequence of polyhedra endowed with simplicial subdivisions with mesh size going to zero, this is not enough to extend the proof to $C$, since the discontinuities of $f$ on the boundary of $C$ prevent us from taking the limit of the sequence of polyhedra. So to resolve this problem, a different approach is needed.

\section{A game-theoretic application}

Discontinuities arise naturally in various fields of study, such as economics and game theory; see, e.g., Arrow and Hahn (1971), Fudenberg and Tirole (1991), and Herings (1996). In this section we give a non-cooperative game-theoretic application and state a sufficient condition under which a Nash equilibrium exists for the case where discontinuities may occur. A non-cooperative game consists of a finite number of players, say, $N$. Player $i$, $i \in I_{N}$, has available a set of actions, denoted by $S^{i}$. For any $i \in I_{N}$, the set $S^{i}$ is an $m_{i}$-dimensional bounded polyhedron in $\mathbb{R}^{n_{i}}$ for some non-negative integers $m_{i}$ and $n_{i}$. The cartesian product $\Pi_{i=1}^{N} S^{i}$ is called the strategy space of the game and is denoted by $S$. An element $x=\left(x_{1}, \cdots, x_{N}\right)$ of $S$ is called a strategy combination. A strategy combination $x \in S$ yields a payoff $u_{i}\left(x_{i}, x_{-i}\right)$ to player $i$, where $x_{-i}=\left(x_{j}\right)_{j \neq i}$ denotes the actions of players other than player $i$ in strategy $x$. A strategy combination $x^{*} \in S$ is a Nash equilibrium if for every player $i \in I_{N}$ action $x_{i}^{*}$ maximizes the payoff of player $i$ given that the other players choose $x_{-i}^{*}$, i.e., for all $i \in I_{N}$

$$
u_{i}\left(x_{i}^{*}, x_{-i}^{*}\right) \geq u_{i}\left(x_{i}, x_{-i}^{*}\right) \text { for all } x_{i} \in S^{i} .
$$

For $x_{-i} \in \prod_{j \neq i} S^{j}$, let $b_{i}\left(x_{-i}\right)$ be a best reply of player $i$ when the other players choose action $x_{-i}$, i.e., $b_{i}\left(x_{-i}\right)$ maximizes $u_{i}\left(x_{i}, x_{-i}\right)$ over $x_{i} \in S^{i}, i \in I_{N}$. Clearly, $x^{*} \in S$ is a Nash 
equilibrium if $b_{i}\left(x_{-i}^{*}\right)=x_{i}^{*}$ for all $i \in I_{N}$. It is well-known that if all best reply functions $b_{i}$, $i \in I_{N}$, are continuous, then there exists a Nash equilibrium, see e.g., Glicksberg (1952). The conditions on the functions $u_{i}$ to guarantee that the best reply functions are continuous are known to be rather strong. Here we show that a Nash equilibrium exists under the following weaker condition on the best reply functions.

Theorem 3.1 Let $S=\prod_{i=1}^{N} S^{i}$ be the cartesian product of $N$ non-empty bounded polyhedra and, for $i \in I_{N}$, let $b_{i}: \Pi_{j \neq i} S^{j} \rightarrow S^{i}$ be best reply functions such that for every $x \in S$ for which $b_{j}\left(x_{-j}\right) \neq x_{j}$ for some $j \in I_{N}$ there exists $\delta>0$ satisfying that for every $y$, $z \in B(x, \delta) \cap S$ we have

$$
\sum_{i=1}^{N}\left(b_{i}\left(y_{-i}\right)-y_{i}\right)^{\top}\left(b_{i}\left(z_{-i}\right)-z_{i}\right) \geq 0 .
$$

Then there exists a point $x^{*} \in S$ satisfying $b_{i}\left(x_{-i}^{*}\right)=x_{i}^{*}$ for all $i \in I_{N}$.

Proof: For $i \in I_{N}$, let $S^{i}$ be an $m_{i}$-dimensional polyhedron in $\mathbb{R}^{n_{i}}$. Define the function $r_{i}$ from $S$ to $\mathbb{R}^{n_{i}}$ by $r_{i}(x)=b_{i}\left(x_{-i}\right)$. The condition in the theorem implies that for all $x \in S$ there exists $\delta>0$ satisfying that for every $y, z \in B(x, \delta) \cap S$ we have

$$
\sum_{i=1}^{N}\left(r_{i}(y)-y_{i}\right)^{\top}\left(r_{i}(z)-z_{i}\right) \geq 0
$$

For $x \in S$, define $r(x)=\left(r_{1}(x), \cdots, r_{N}(x)\right)$. Clearly, the set $S$ is an $m$-dimensional bounded polyhedron in $\mathbb{R}^{n}$, with $m=\sum_{j=1}^{N} m_{j}$ and $n=\sum_{j=1}^{N} n_{j}$. Then $r$ is a function from $S$ into itself and for every $x \in S$ for which $r(x) \neq x$ there exists $\delta>0$ satisfying that for every $y$, $z \in B(x, \delta) \cap S$ we have

$$
(r(y)-y)^{\top}(r(z)-z) \geq 0
$$

i.e., the function $r$ satisfies the conditions of Theorem 2.2 with $P$ equal to $S$. Hence, the function $r$ has a fixed point $x^{*}$ on $S$, i.e., $r\left(x^{*}\right)=x^{*}$. Clearly, a fixed point $x^{*}$ of $r$ satisfies $b_{i}\left(x_{-i}^{*}\right)=x_{i}^{*}$ for all $i \in I_{N}$.

Notice that if all functions $b_{i}$ are continuous, then the conditions in Theorem 3.1 are automatically satisfied. Indeed, if $b_{j}\left(x_{-j}\right) \neq x_{j}$ for some $j \in I_{N}$, then it holds that

$$
\sum_{i=1}^{N}\left(b_{i}\left(x_{-i}\right)-x_{i}\right)^{\top}\left(b_{i}\left(x_{-i}\right)-x_{i}\right)>0 .
$$

Hence, for such an $x \in S$ there exists $\delta>0$ satisfying that for every $y, z \in B(x, \delta) \cap S$

$$
\sum_{i=1}^{N}\left(b_{i}\left(y_{-i}\right)-y_{i}\right)^{\top}\left(b_{i}\left(z_{-i}\right)-z_{i}\right) \geq 0 \text {. }
$$




\section{References}

[1] E.L. Allgower and K. Georg (1990), Numerical Continuation Methods: An Introduction, Springer, Berlin.

[2] K.J. Arrow and F.H. Hahn (1971), General Competitive Analysis, Holden-Day, San Francisco.

[3] L.E.J. Brouwer (1912), "Über Abbildung von Mannigfaltigkeiten," Mathematische Annalen (71) 97-115.

[4] J. Caristi (1976), "Fixed point theorems for mappings satisfying inwardness conditions," Transaction of American Mathematical Society (215), 241-251.

[5] I.L. Glicksberg (1952), "A further generalization of the Kakutani fixed point theorem with application to Nash equilibrium points," Proceedings of the National Academy of Sciences (38), 170-174.

[6] P.J.J. Herings (1996), Static and Dynamic Aspects of General Disequilibrium Theory, Kluwer, Boston.

[7] V.I. Istratescu (1981), Fixed Point Theory: An Introduction, Reidel Publishing, Dordrecht.

[8] S. Kakutani (1941), "A generalization of Brouwer's fixed point theorem," Duke Mathematical Journal (8), 457-459.

[9] H.E. Scarf (1973), The Computation of Economic Equilibria, Yale University Press, New Haven.

[10] J. Schauder (1930), "Der Fixpunktsatz in Funktionalraümen," Studia Math. (2), 171180.

[11] J.B. Shoven and J. Whalley (1992), Applying General Equilibrium, Cambridge University Press, New York.

[12] R. Smart (1974), Fixed Point Theorems, Cambridge University Press, Cambridge.

[13] A.J.J. Talman and Y. Yamamoto (1989), "A simplicial algorithm for stationary point problems on polytopes," Mathematics of Operations Research (14), 383-399.

[14] A. Tarski (1955), "A lattice-theoretical fixed point theorem and its applications," Pacific Journal of Mathematics (5), 285-309. 
[15] M.J. Todd (1976), The Computation of Fixed Points and Applications, Springer, Berlin.

[16] Z. Yang (1999), Computing Equilibria and Fixed Points, Kluwer, Boston.

[17] Z. Yang (2004), Discrete fixed point analysis and its applications, FBA Discussion paper 210, Yokohama National University, Yokohama. 